\title{
Recrutamento de sujeitos em ensaios clínicos multicêntricos internacionais financiados pela indústria'
}

\section{Recruitment of subjects in industry-sponsored multicenter international clinical trials}

\section{Rodrigo Batagello}

Pontifícia Universidade Católica do Paraná. Programa de PósGraduação em Bioética. Curitiba, PR, Brasil.

E-mail: rodrigo.batagelloळgmail.com

\section{Correspondência}

Av. Miguel Petroni, 4.900, Jardim Bandeirantes. São Carlos, SP, Brasil. CEP $13563-470$.

\section{Resumo}

Este estudo trata do recrutamento de sujeitos de pesquisa em ensaios clínicos multicêntricos internacionais financiados pela indústria executados em uma universidade pública brasileira entre janeiro de 2010 e dezembro de 2016. Analisou-se um conjunto de 63 contratos de ensaios clínicos, documentos administrativos e regulatórios. Os resultados demonstraram a participação de 75 países, dos quais 16\% são latino-americanos. Foram obtidos dados sobre o recrutamento de sujeitos de pesquisa em âmbito local (356 sujeitos), nacional (3.774 sujeitos) e internacional (82.696 sujeitos), além de informações sobre o número de centros participantes no Brasil e o custo de cada ensaio. A maioria dos ensaios era de fase III (77,78\% dos casos) e previa um recrutamento total nacional entre $18 \mathrm{e}$ 8 o sujeitos de pesquisa por ensaio (entre $4,42 \%$ e $11,46 \%$ do recrutamento internacional). Identificouse forte correlação negativa entre os dados sobre contratos, orçamento e recrutamento e a cotação média anual do dólar. A totalidade dos ensaios adotou método competitivo de recrutamento associado ao pagamento por sujeito incluído na pesquisa. Isso é preocupante e demonstra uma contradição entre essa prática e as recomendações das diretrizes éticas nacionais. A ausência da informação sobre o número de sujeitos a serem recrutados no país revela, em $19 \%$ dos casos, um erro recorrente no processo regulatório. Conclui-se que a disponibilidade de informações detalhadas sobre o recrutamento nas pesquisas envolvendo seres humanos é importante

1 A pesquisa foi realizada com o apoio de bolsa de estudo concedida pelo Programa Nacional de Pós-Doutorado da Coordenação de Aperfeiçoamento de Pessoal de Nível Superior (Capes). 
para o correto dimensionamento e organização dos esforços de proteção dos sujeitos de pesquisa, e que atualmente essa premissa não está sendo cumprida de modo adequado.

Palavras-chave: Recrutamento de Sujeitos de Pesquisa; Ensaio Clínico; Estudos Multicêntricos; Cooperação Internacional; Sujeitos da Pesquisa.

\section{Abstract}

This study concerns the recruitment of research subjects in industry-sponsored multicentric international clinical trials carried out at a Brazilian public university between January 2010 and December 2016. A set of 63 clinical trial agreements, administrative and regulatory documents was analyzed. The results showed that 75 countries were involved in these international trials, of which $16 \%$ were Latin American. Data about the recruitment of research subjects at the local (356 subjects), national (3774 subjects) and international (82,696 subjects) levels were obtained, as well as information on the number of Brazilian research centers involved and the costs of each trial. Phase III trials were the most frequent ( $77.78 \%$ of the cases) and they estimated a total national enrollment between 18 and 80 research subjects per trial (which means between $4.42 \%$ and $11.46 \%$ of international recruitment). A negative correlation was found between agreements, research resources, recruitment data, and yearly average dollar exchange rate. All the trials adopted competitive recruitment method associated with payment for subject included in the research. This is worrying and shows contradiction between this practice and the recommended national ethical guidelines. The lack of data on recruitment at the national level reveals, in $19 \%$ of the cases, a frequent regulatory process failure. It is concluded that the availability of detailed data on recruitment for researches involving human beings is important for the accurate sizing and organization of the efforts to protect research subjects, and that currently this premise is not being appropriately followed.

Keywords: Patient Selection; Clinical Trial; Multicenter Study; International Cooperation; Research Subjects.

\section{Introdução}

Os ensaios clínicos multicêntricos internacionais e financiados pela indústria (Mifi) são o modelo predominante nas pesquisas de desenvolvimento de novas drogas para a saúde humana (Atal et al., 2015; Flacco et al., 2015; Ivanov et al., 2017). Por isso, esse modelo de organização do trabalho científico é um dos principais objetos dos esforços regulatórios em todo o mundo.

Apesar da existência de inúmeras diretrizes regulatórias e das plataformas de registro de ensaios clínicos, ainda é uma tarefa complicada conhecer a distribuição dos sujeitos de pesquisa entre os diferentes centros de pesquisa espalhados pelo mundo - embora o número de sujeitos participantes em um ensaio clínico seja definido por critérios estatísticos, sendo número bastante preciso (Hackshaw, 2009). Essa dificuldade existe pelo fato de não ser exigido dos ensaios Mifi tal nível de detalhamento em nenhum dos documentos regulatórios internacionais e, principalmente, de não ser demandado em nenhuma das plataformas de registro de ensaios clínicos (Dal-Ré et al., 2011).

Na plataforma ClinicalTrials.gov, por exemplo, a única informação exigida é a estimativa total do número de sujeitos que serão recrutados (estimated enrollment). No caso do Registro Brasileiro de Ensaios Clínicos (ReBEC) e da plataforma de registro secundário da Organização Mundial da Saúde, o International Clinical Trials Registry Platform (ICTRP), os campos são idênticos: tamanho da amostra alvo (target sample size).

Porém, mesmo quando se trata de identificar o número total de sujeitos recrutados para um determinado ensaio multicêntrico, encontram-se alguns obstáculos. O mais frequente é consequência do registro de um mesmo ensaio clínico multicêntrico internacional em várias plataformas primárias, afinal, cada país pode manter sua própria plataforma. Isso produz entradas múltiplas de dados sobre um mesmo protocolo de pesquisa, o que pode produzir informação ambígua. O protocolo número CINC424A2401 é um exemplo dessa situação: de acordo com o ICTRP, este foi registrado 14 vezes em diferentes plataformas primárias. Em um registro, o número de sujeitos não foi preenchido, e nos demais 
foram encontrados seis valores distintos: três vezes o valor de 950, três vezes de 2.500, três de 2.484, duas de 1.600 sujeitos, um registro indicando 991 e um com 2.232 sujeitos participantes.

Esse problema envolvendo as plataformas de registros clínicos já foi notado por outros autores (Viergever et al., 2014). A informação atualmente disponível sobre o recrutamento de sujeitos de pesquisa é frágil e, em certas situações, não é suficiente para a compreensão efetiva da organização das redes de recrutamento dos ensaios Mifi (Dal-Ré et al., 2011). 0 conhecimento detalhado da dinâmica de recrutamento dos sujeitos de pesquisa em cada país participante é fundamental para o planejamento das ações e políticas regulatórias, para a coordenação dos sistemas de saúde e para as avaliações dos benefícios e riscos decorrentes do envolvimento de uma determinada população nos ensaios clínicos Mifi. Afinal, o número de sujeitos expostos às drogas experimentais em um determinado país deveria ser uma informação prioritária para a organização de suas estruturas regulatórias e o monitoramento de seu sistema de saúde.

O objetivo deste estudo é apresentar uma descrição detalhada do recrutamento de sujeitos de pesquisa em uma universidade pública brasileira e discutir aspectos dessa atividade no país. Para isso, foi utilizado como fonte de dados um conjunto de contratos de ensaios clínicos e outros documentos produzidos durante os processos administrativo e regulatório, necessários para a execução de ensaios clínicos randomizados e controlados Mifi em uma universidade pública do estado de São Paulo.

\section{Método}

Os dados foram obtidos a partir da análise de um conjunto de contratos de ensaios clínicos e seus respectivos documentos produzidos durante o processo regulatório e administrativo, necessários para a realização de ensaios clínicos Mifi em uma universidade pública do estado de São Paulo, Brasil.

Os documentos coletados fazem parte do que as Diretrizes para a boa prática clínica $E 6$ ( $R 1)$ denominam documentos essenciais para a condução de um ensaio clínico (essential documents for the conduct of a Clinical Trial) (ICH, 1996). São eles: o contrato de ensaio clínico Mifi assinado e datado, o parecer da Comissão Nacional de Ética em Pesquisa do Ministério da Saúde brasileiro (Conep/MS), o parecer do Comitê de Ética em Pesquisa (CEP), o parecer científico e o parecer jurídico, esses últimos produzidos pelas instâncias acadêmicoadministrativas da universidade que participou dos ensaios clínicos.

Esses documentos foram coletados no arquivo da instituição em questão entre setembro de 2016 e janeiro de 2017. Os contratos de ensaios clínicos foram assinados entre janeiro de 2010 e dezembro de 2016 e representam a totalidade dos documentos disponíveis no arquivo da instituição durante a coleta.

Para a organização cronológica dos dados apresentados e dos documentos analisados, e para a consulta da taxa de câmbio e conversão dos valores em real para o dólar americano, foram utilizadas as datas de assinatura dos respectivos contratos de ensaios clínicos.

Como o objeto desses contratos era a execução de um protocolo de ensaio clínico Mifi, os dados obtidos através desses documentos dizem respeito à totalidade do estudo e permitiram descrever as características dos ensaios clínicos Mifi em três âmbitos: o local (ou o centro), o nacional e o internacional, embora nem todas as informações recolhidas estivessem disponíveis para todos eles.

Foram localizados os dados sobre o recrutamento de sujeitos de pesquisa nos três âmbitos. Os dados sobre o número de centros de pesquisa envolvidos nas pesquisas são nacionais e os dados sobre os valores pagos por sujeito incluído em cada um dos ensaios são locais, pois tratam apenas dos valores repassados pelos patrocinadores à universidade que executou os ensaios clínicos.

Para a coleta foi desenvolvido um banco de dados composto de 21 campos. Foi conduzida uma análise estatística descritiva desses dados e, quando necessário, foi utilizado o software $\mathrm{JASP}^{2}$ para a condução dos testes.

2 JASP TEAM. JASP [Software]. Version o.8.6. Amsterdam: University of Amsterdam, 2018. Disponível em: <https://jasp-stats.org/>. Acesso em: 10 jan. 2017. 
Em função de alguns dos resultados obtidos, formulou-se a hipótese de que a distribuição anual dos contratos, do total de sujeitos e do total de recursos poderia estar correlacionada com a variação cambial do dólar americano. Para explorar essa hipótese, decidiu-se medir o grau de correlação entre as variáveis de interesse e a taxa de câmbio por intermédio do coeficiente de correlação de postos de Spearman.

Para a execução das medidas de correlação, foram estruturadas as seguintes matrizes: (1) distribuição anual do número de contratos assinados pelo centro versus a cotação média anual do dólar; (2) distribuição anual do orçamento versus a cotação média anual do dólar; (3) distribuição anual do número de sujeitos previstos para recrutamento no centro versus a cotação média anual do dólar. Os dados foram ordenados no sentido decrescente e os pares ordenados foram testados. A taxa de câmbio utilizada para a construção do ranque da moeda americana foi a média anual do dólar comercial para contabilidade divulgada pelo Instituto de Pesquisas Econômicas Aplicadas (Ipea).

\section{Resultados}

No período entre janeiro de 2010 e dezembro de 2016, a universidade brasileira estudada assinou 63 contratos para a execução de ensaios clínicos multicêntricos internacionais financiados pela indústria. Esses contratos e os respectivos documentos foram incluídos na amostra $(n=63)$.

Esses ensaios clínicos eram majoritariamente de fase III ( $77,78 \%$ dos casos) e, no total, foram desenvolvidos em centros de pesquisa localizados em 75 países diferentes.

No plano internacional, os 63 ensaios clínicos recrutaram um total de 82.696 sujeitos em todo o mundo. Como demonstra a Figura 1, a maioria dos protocolos de pesquisa tinha como meta de recrutamento um número que variou entre 239, no primeiro quartil, e 1.28o, no terceiro quartil, de sujeitos no mundo. 0 valor mínimo apurado foi de 37 sujeitos, a mediana foi de 637 , e o maior valor identificado foi de 9.340 sujeitos recrutados. Em 87,30\% das amostras ( $n=63)$, a previsão total de recrutamento foi inferior a 2 mil sujeitos. Metas de recrutamentos superiores a 4 mil sujeitos foram identificados em apenas $11,11 \%$ dos registros avaliados.
No âmbito nacional, em 12 casos (19,05\% dos registros) não foi possível determinar a quantidade de sujeitos a serem recrutados no Brasil. Nos registros que continham a informação sobre recrutamento no país $(n=51)$, apurou-se o total de 3.774 sujeitos.

Ainda sobre os dados nacionais, como demonstra a Figura 1, o valor máximo observado foi de 690 sujeitos recrutados para um único ensaio, e o valor mínimo foi de dois participantes. 0 primeiro quartil foi de 18 sujeitos, o valor da mediana foi de $45 \mathrm{e}$ do terceiro quartil de 8 o sujeitos. Em $86,28 \%$ das amostras analisadas, a previsão de recrutamento foi igual ou inferior a 100 sujeitos de pesquisa. Em apenas sete ensaios clínicos a meta de recrutamento no país foi superior a 100 pacientes.

Em relação aos dados do centro, não foi possível determinar o número de sujeitos recrutados em cinco registros $(7,94 \%)$. A informação sobre o recrutamento estava disponível em 58 registros (92,06\%), que previam a participação total de 356 sujeitos de pesquisa.

As medidas de posição foram as seguintes: o valor mínimo foi um único sujeito, o primeiro quartil foram três, a mediana foram cinco, o terceiro quartil foram nove sujeitos e o valor máximo observado foram 20 pacientes. Em 51 desses registros (87,93\%), a previsão era recrutar um número menor ou igual a dez sujeitos (Figura 1). Em apenas sete registros, ou 12,07\% dos ensaios, a previsão de recrutamento foi superior a dez.

Para estabelecer uma relação de proporcionalidade entre os diferentes estratos de recrutamento, optouse por analisar separadamente os 49 registros de ensaios clínicos Mifi que continham dados nos âmbitos internacional, nacional e local. Para esses registros, os números totais de recrutamento foram: 58.303 internacionalmente, 3.678 no Brasil $(6,31 \%$ dos sujeitos) e 323 no centro (o, $55 \%$ do total internacional e $8,78 \%$ do total nacional).

Em relação à participação proporcional brasileira no recrutamento de sujeitos de pesquisa nos ensaios Mifi individualmente, foram obtidas as seguintes medidas de posição: a proporção mínima foi de o,69\%, o primeiro quartil foi $4,42 \%$, a mediana foi $7,04 \%$, o terceiro quartil $11,46 \%$ e a máxima de $29,41 \%$. Em apenas cinco registros (10,20\% dos ensaios) a participação brasileira ultrapassou 16\% do número total de sujeitos recrutados internacionalmente. 
Figura I - Distribuição internacional, nacional e local (no centro) do número de sujeitos previstos para recrutamento nos 63 ensaios clínicos multicêntricos internacionais financiados pela indústria alvo da pesquisa

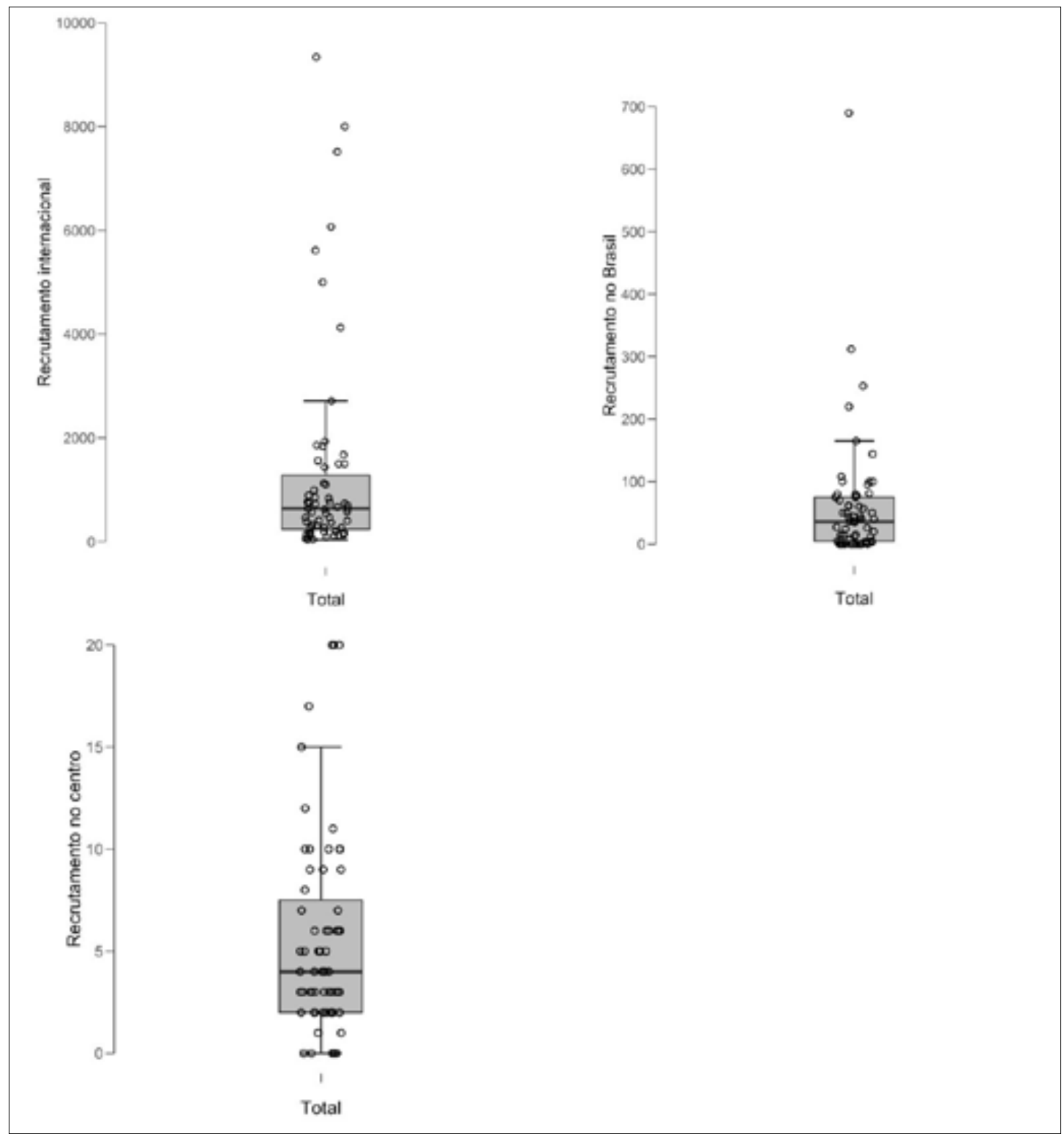

As medidas de posição dos dados relativos ao centro foram as seguintes: a proporção mínima observada foi de $0,07 \%$ do total internacional e $2,67 \%$ do total nacional; o primeiro quartil foi o, $47 \%$ e $7,69 \%$ dos totais internacional e nacional, respectivamente; a mediana encontrada foi de $0,81 \%$ e $12,50 \%$, respectivamente; o terceiro quartil foi de $1,94 \%$ e $20 \%$ dos respectivos totais; o valor máximo observado foi de $5,43 \%$ do total internacional e $100 \%$ dos sujeitos recrutados no Brasil. Em apenas três casos o número de participantes recrutados no centro de pesquisa ultrapassou 3,5\% do total mundial.

Os registros de ensaios clínicos Mifi permitiram conhecer a dimensão das redes multicêntricas que se estabeleceram no Brasil para o recrutamento de sujeitos e execução dos protocolos de pesquisa. Foi possível dimensionar as redes de centros brasileiros pelo levantamento do número de centros 
participantes em cada ensaio, como demonstra a Figura 2. Essa informação foi encontrada em todos os registros da amostra $(n=63)$.

A participação de um número igual ou menor a 19 centros na execução de um único ensaio clínico Mifi foi observada em 57 registros, o que representa 90,48\% dos registros da amostra. Foram identificados cinco registros $(7,94 \%$ da amostra) com centro único no Brasil. Ensaios multicêntricos que envolveram cinco centros participantes foram os mais frequentes, com 12 registros $(19,05 \%)$.

\section{Figura 2 - Dimensão da rede de recrutamento dos 63 ensaios clínicos multicêntricos internacionais financiados pela indústria conforme o número de centros de pesquisa brasileiros participantes}

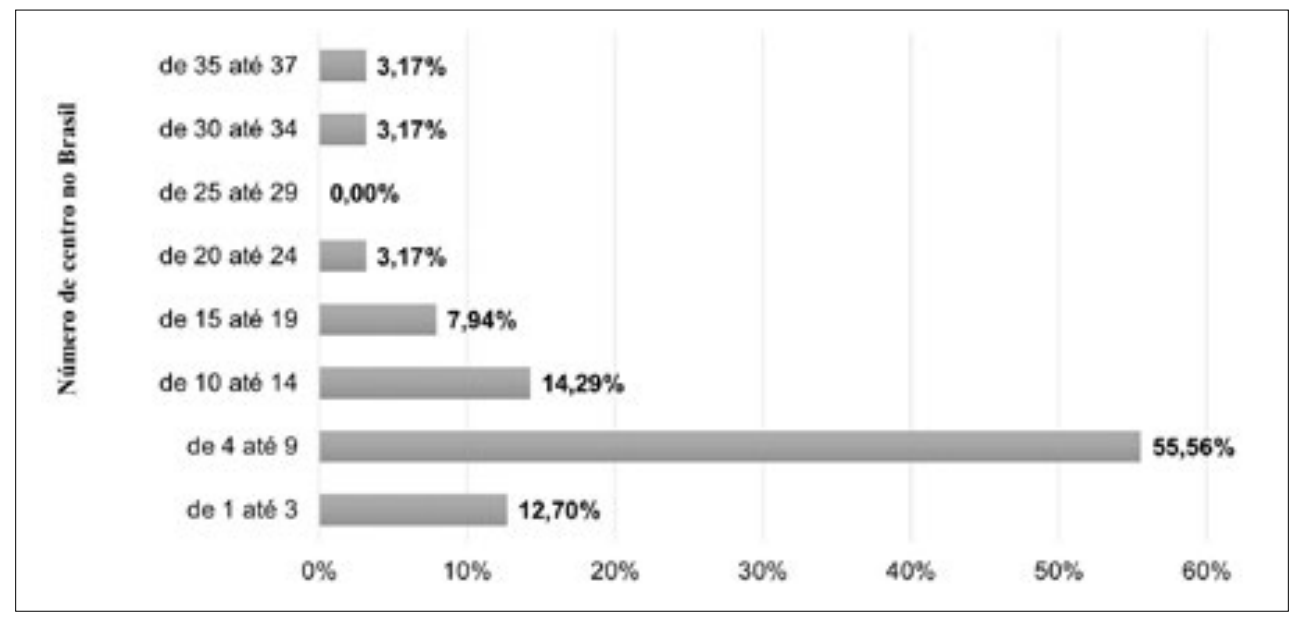

Constatou-se que os orçamentos dos ensaios clínicos Mifi são elaborados a partir de um valor predefinido por sujeito de pesquisa recrutado e mantido no estudo até sua conclusão. Os documentos analisados são anteriores ao início da pesquisa, portanto os valores apresentados assumem como pressuposto que o centro recrutou todos os sujeitos e que estes concluirão todas as etapas previstas nos protocolos.

Em cinco casos não foi possível determinar o número de sujeitos que seriam recrutados pelo centro, consequentemente não foi possível determinar o valor investido por sujeito incluído nesses contratos. Como se pode observar na Figura 3, em 38 contratos de ensaios clínicos (ou 65,52\% da amostra), o valor dispensado por sujeito incluído foi igual ou inferior a US\$ 15 mil.

As medidas de posição encontradas foram: o valor mínimo foi de US\$2 244,36 , o primeiro quartil foi de US\$ 5.605,52, a mediana de US\$ 9.773,33 e o terceiro quartil foi de US\$21.180,oo, pagos ao centro por sujeito recrutado e acompanhado. Acima dos US\$ 36 mil foram identificadas apenas duas ocorrências, com os respectivos valores de US\$ 52.416, oo e US\$ $58.845,90$ por sujeito recrutado, sendo esse último o valor máximo apurado.

A Tabela 1 demonstra a distribuição anual dos ensaios clínicos Mifi, dos recursos totais pagos ao centro de pesquisa alvo do estudo e da previsão de recrutamento dos pacientes no Brasil e no centro.

Os contratos foram reunidos por ano em função das respectivas datas de assinatura. 0 total dos recursos pagos ao centro foi obtido primeiramente pela multiplicação do número de sujeitos previstos pelo valor estabelecido em contrato para cada sujeito e, em seguida, pela soma dos valores totais dos contratos reunidos nos anos correspondentes. Embora a informação sobre o número de sujeitos a serem recrutados no centro de pesquisa não tenha sido localizada em cinco casos, nos documentos analisados havia a informação do valor total que seria repassado ao centro. Em apenas um caso, de 2013, não foi possível localizar nenhuma informação sobre o orçamento. 
Figura 3 - Distribuição dos 58 contratos de ensaios clínicos multicêntricos internacionais e financiados pela indústria em função do valor pago ao centro para cada sujeito incluído

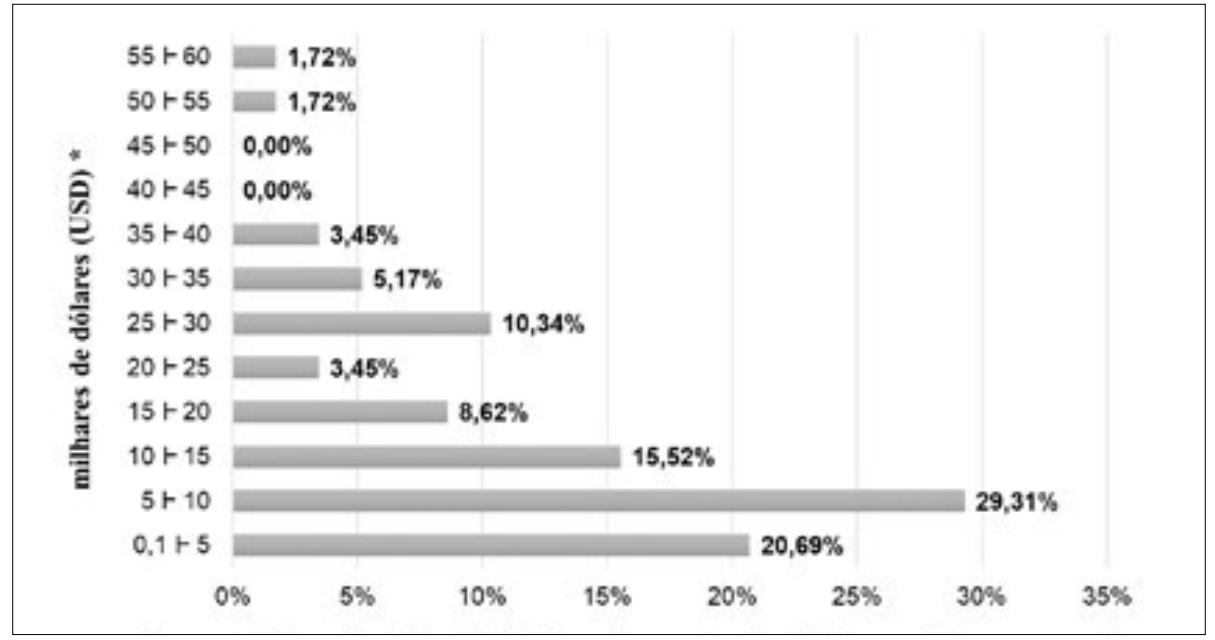

* A conversão dos valores foi realizada de acordo com a taxa do câmbio comercial para compra do dólar apurada na data de assinatura dos respectivos contratos. Fonte: Ipeadata ${ }^{3}$

Tabela I - Distribuição anual dos contratos de ensaios clínicos dos sujeitos de pesquisa recrutados no Brasil e no centro entre 2010 e 2016

\begin{tabular}{|c|c|c|c|c|c|c|c|c|}
\hline \multirow{2}{*}{ Ano } & \multirow{2}{*}{ Contratos } & \multirow{2}{*}{$\%$} & \multirow{2}{*}{$\begin{array}{l}\text { Orçamento } \\
\text { (US\$)**** }\end{array}$} & \multirow{2}{*}{$\%$} & \multicolumn{4}{|c|}{ Sujeitos recrutados } \\
\hline & & & & & Brasil & $\%$ & Centro & $\%$ \\
\hline 2010 & 16 & 25,40 & $778.423,28$ & 18,34 & $836^{* *}$ & 22,15 & $|2|^{* * *}$ & 33,99 \\
\hline 2011 & 20 & 31,75 & $1.965 .784,57$ & 46,32 & $1708^{* *}$ & 45,26 & 131 & 36,80 \\
\hline 2012 & 11 & 17,46 & $627,823,67$ & 14,79 & $396^{* *}$ & 10,49 & $50^{* * *}$ & 14,04 \\
\hline 2013 & 6 & 9,52 & $419.152,44^{*}$ & 9,88 & $249^{* *}$ & 6,60 & $15^{* * *}$ & 4,21 \\
\hline 2014 & 5 & 7,94 & $375.408,49$ & 8,84 & 508 & 13,46 & $29^{* * *}$ & 8,15 \\
\hline 2015 & 0 & 0 & 0 & 0 & 0 & 0 & 0 & 0 \\
\hline 2016 & 5 & 7,94 & $77 \cdot 746,68$ & 1,83 & $77^{* *}$ & 2,04 & $10^{* *}$ & 2,81 \\
\hline Total & 63 & 100 & $4.244 .339,13$ & 100 & 3774 & 100 & 356 & 100 \\
\hline
\end{tabular}

* Não foi possível encontrar a informação sobre o orçamento de um contrato em 2013.

** O número de sujeitos recrutados no país não foi localizado em doze casos, quatro contratos de 2010, um de 2011, dois de 2012 , dois de 2013 e três de 2016 .

*** 0 número de sujeitos recrutados no centro não foi localizado em cinco casos, um em cada ano marcado.

**** Taxa do câmbio comercial para compra do dólar apurada na data da assinatura do contrato.

Fonte: Ipeadata ${ }^{4}$

Observou-se uma significativa variação anual na distribuição dos dados, com destaque para a marcante redução no número de ensaios clínicos Mifi desenvolvidos no centro entre 2011 e 2015. No período analisado, o ano de 2015 foi o único no qual não foi assinado nenhum contrato.

Foi preciso formular uma explicação e identificar possíveis variáveis que pudessem auxiliar na 
compreensão dessa redução no número de contratos a partir de 2012 e que culminou com a completa ausência de contratos de ensaios clínicos Mifi no ano de 2015 .

Uma das hipóteses plausíveis seria considerar a influência da oscilação da taxa de câmbio do dólar americano. Afinal, compreendida como um empreendimento industrial, a atividade de pesquisa e o desenvolvimento de novas drogas, realizados em âmbito internacional, está sujeita aos impactos da flutuação do mercado cambial (KPMG LLP, 2016). Do ponto de vista dos países participantes, o mercado cambial pode ser visto como um elemento que afeta diretamente sua competitividade (KPMG LLP, 2016).

Para testar essa hipótese, decidiu-se medir a correlação entre alguns dos dados encontrados com o coeficiente de correlação de postos de Spearman e avaliar a força dessa correlação entre os dados anuais obtidos e a taxa de câmbio anual média da moeda americana no mercado brasileiro.

Tabela 2 - Medidas do coeficiente de correlação de
postos de Spearman entre a distribuição anual dos
contratos de ensaios clínicos, dos orçamentos totais e
dos sujeitos recrutados no centro de pesquisa e a cotação
média anual do dólar americano entre 2010 e 2016

Todos os testes realizados foram unicaudais para correlação negativa e com nível de significância $\alpha=5 \%$. Taxa média anual do câmbio do dólar comercial para registro contábil.

Fonte: Ipeadata ${ }^{5}$
Como demonstra a Tabela 2, em todos os casos foi identificada uma forte correlação negativa entre as variáveis: a valorização da moeda americana se associou fortemente aos números decrescentes de sujeitos recrutados no centro, de contratos firmados pelo centro e de recursos recebidos pelo centro para a execução de ensaios clínicos Mifi entre 2010 e 2016. Esses resultados permitem aceitar a hipótese de que existe uma associação importante entre os dados obtidos nos documentos e as taxas de câmbio.

Embora o objetivo da pesquisa não tenha previsto uma análise qualitativa do conteúdo dos contratos, um item chamou a atenção e se tornou um resultado corolário: todos os contratos analisados indicavam que o processo de recrutamento adotado seria o competitivo e que o pagamento ao centro se vincularia ao número de sujeitos incluídos nas pesquisas. Os contratos estabeleceram uma meta mínima de recrutamento que o centro deveria atingir e, em alguns casos, a indicação de um prazo ou uma data limite para que essa meta fosse atingida.

\section{Discussão}

Os dados apresentados permitem estabelecer um cenário sobre o envolvimento de centros de pesquisa e pacientes brasileiros em pesquisas clínicas Mifi e revelam algumas características dessa participação que podem ser comuns a outros países, particularmente aos latino-americanos, uma vez que existem muitas semelhanças entre estes e vários são destinos frequentes de ensaios Mifi (Atal et al., 2015). A América Latina e o Caribe tiveram uma representação significativa nos dados, concentrando $16 \%$ do total de países envolvidos nos ensaios clínicos.

Os artigos encontrados sobre o recrutamento de sujeitos de pesquisa em ensaios Mifi apresentam alguns números próximos dos obtidos, especialmente em relação ao custo por sujeito incluído nos ensaios (Berndt; Cockburn, 2014). Porém, outros trabalhos apontam número um pouco distinto, principalmente em relação ao número de sujeitos recrutados nos âmbitos nacional e local (Murthy; Mandl; Bourgeois, 
2015). Muitos fatores podem contribuir para a variação das medidas, mas a diversidade de fontes de dados pode determinar essa variação. De fato, a imprecisão dessas informações nas plataformas de registro de ensaios clínicos exige dos pesquisadores recorrer a fontes nem sempre precisas e atualizadas (Van Valkenhoef et al., 2012; Viergever et al., 2014; Wood, 2009).

Soma-se a esse problema a característica dinâmica dos ensaios clínicos como atividade científica e comercial, que está em permanente transformação. Segundo alguns autores, ao longo das duas últimas décadas, a configuração dos ensaios clínicos sofreu alterações em sua estrutura logística: o número de centros participantes e o custo por sujeito incluído aumentaram, o número médio de sujeitos alocados em cada centro foi reduzido e o trabalho de cada um dos centros se tornou mais intenso e complexo (Berndt; Cockburn, 2014).

Também é preciso compreender os fatores comerciais que podem favorecer ou dificultar a inserção de um determinado país ou região nessa rede dos ensaios clínicos Mifi.

Como foi demostrado, identificou-se uma forte correlação negativa entre os dados testados e a taxa de câmbio anual, mas essa informação contraria a regra que pressupõe que a moeda nacional desvalorizada ante o dólar aumentaria a competitividade do país (KPMG LLP, 2016).

Embora o coeficiente de correlação não meça ou represente uma relação de causa-efeito, ele demonstra que os valores mais altos na cotação média anual do dólar estiveram associados aos valores anuais menores da distribuição dos dados, quando teoricamente deveria ocorrer o oposto (KPMG LLP, 2016).

É necessário destacar, contudo, que a desvalorização da moeda brasileira ante a moeda americana a partir de 2012 teve relação direta com a instabilidade política e a crise financeira que se abateu sobre o país nesse período, sobretudo a partir de 2014 (Bresser-Pereira, 2017). Portanto, a explicação para a redução do número de contratos de ensaios clínicos pode ter relação com esse contexto de instabilidade, do qual a taxa de câmbio é um dos principais termômetros. Ao conduzir um ensaio clínico, a instituição assume um compromisso que, de acordo com a maioria dos contratos, é de aproximadamente 60 meses. Um contexto de incerteza pode dissuadir o patrocinador a optar pelo país, uma vez que pode representar riscos para etapas sensíveis como o recrutamento e a adesão dos sujeitos de pesquisa ao protocolo.

O recrutamento e a manutenção de sujeitos da pesquisa são etapas críticas para a condução dos ensaios clínicos e têm relação direta com sua própria viabilidade científica e financeira (Dal-Ré et al., 2011; Kost et al., 2015). Afinal, é preciso atender aos parâmetros estatísticos de amostragem necessários para a demonstração da validade dos resultados (Watson; Torgerson, 2006) que fundamentarão o licenciamento comercial das novas drogas, o que explica as inúmeras técnicas e estratégias para alcançar as metas de recrutamento e garantir a adesão e a manutenção dos sujeitos ao protocolo (Gupta et al., 2015; McDonald et al., 2011; Treweek et al., 2013). Um contexto de severa instabilidade política representa um risco para o patrocinador e pode justificar a correlação negativa identificada entre os dados e a taxa de câmbio.

De qualquer maneira, o ponto relevante que essa discussão apresenta é a necessidade de levantamentos sistemáticos de informações sobre o processo de recrutamento de sujeitos de pesquisa no Brasil e nos diferentes centros de pesquisa espalhados pelo país. Elas são fundamentais por diversos motivos: do ponto de vista do planejamento de investimentos em pesquisa e desenvolvimento em saúde, é estratégico conhecer quem são e como se comportam os principais atores (públicos e privados) do setor em relação à mobilização de sujeitos de pesquisa e os valores que despendem. Considera-se, portanto, que os resultados deste estudo são um primeiro passo nesse sentido e uma referência para levantamentos futuros.

Ainda do ponto de vista do planejamento, as informações sobre o recrutamento de sujeitos de pesquisa permitem desenvolver índices de desempenho científico que levem em conta a frequência das falhas de recrutamento nas pesquisas, suas consequências médicas e científicas e os custos associados a tais falhas (Dal-Ré et al., 2011; Kost et al., 2015). Todas essas análises são ainda mais necessárias no contexto dos países em 
desenvolvimento, nos quais a escassez de recursos exige uma aplicação eficaz.

No plano regulatório, a disponibilidade de informações sobre o processo de recrutamento nas pesquisas envolvendo seres humanos são importantes para o correto dimensionamento e organização dos esforços de proteção dos sujeitos de pesquisa. Atualmente, no Brasil, não é possível saber, por exemplo, quantos sujeitos de pesquisa um determinado Comitê de Ética acompanha simultaneamente, impossibilitando a avaliação da qualidade desse acompanhamento, nem mesmo da real capacidade dos comitês de realizá-lo.

Além disso, a constatação de que a totalidade dos registros adotou o método competitivo de recrutamento, associado ao pagamento condicionado ao número de sujeitos incluídos, é preocupante e demonstra uma contradição entre essa prática e as recomendações das diretrizes éticas nacionais. De acordo com o item IV.1.p da Resolução do Conselho Nacional de Saúde nº 251/1997, que define as normas de pesquisa envolvendo seres humanos para a área temática de pesquisa com novos fármacos, medicamentos, vacinas e testes diagnósticos, o "financiamento da pesquisa não deve estar vinculado ao pagamento per capita dos sujeitos efetivamente recrutados" (Brasil, 2007). Ao vetar essa modalidade de pagamento, diretamente relacionada à dinâmica de recrutamento, a resolução proíbe a adoção do método competitivo de recrutamento - portanto afirma-se que sua prática no Brasil contraria as diretrizes éticas nacionais vigentes.

Outro dado pertinente ao processo regulatório são as falhas computadas na disponibilidade da informação sobre o número de sujeitos a serem recrutados no Brasil e no centro de pesquisa: respectivamente de 19,05\% e 7,94\%. Esses dados revelam um erro recorrente que perpassa um conjunto de documentos centrais no processo regulatório, em particular os pareceres da Comissão Nacional de Ética em Pesquisa do Ministério da Saúde e do Comitê de Ética em Pesquisa da instituição. 0 fato de o número de sujeitos a serem recrutados no centro de pesquisa e principalmente no país não estar registrado em nenhum dos documentos analisados - e também de não estar publicamente disponível em nenhuma plataforma digital ou banco de dados
(ClinicalTrials.gov, ReBEC ou Plataforma Brasil) indica que o sistema regulatório e os procedimentos institucionais não adotam critérios mínimos de registro para informações que são básicas para a manutenção e avaliação do processo regulatório institucional e, mais importante, nacional.

Tanto as questões de planejamento como as regulatórias são ainda mais relevantes quando se atenta para o fato de que, no Brasil, a maior parte das pesquisas clínicas é conduzida em hospitais universitários públicos e, portanto, envolve pacientes e estruturas do Sistema Único de Saúde (Paschoale et al., 2010), como é o caso do centro que foi objeto deste estudo.

Dados detalhados sobre o recrutamento de sujeitos de pesquisa no Sistema Único de Saúde são necessários para que se possa avaliar a repercussão dessa participação sob diferentes perspectivas: sua relação entre os custos e benefícios; o controle e o monitoramento de eventos adversos graves e custos associados; possíveis contradições entre a disponibilidade de recursos para atendimento e recursos mobilizados para as pesquisas. Portanto, é preciso avançar na elaboração de novos estudos, na construção de bases de dados e no conhecimento sobre esse tema no país.

\section{Referências}

ATAL, I. et al. Differential globalization of industry- and non-industry-sponsored clinical trials. PLoS One, San Francisco, v. 10, n. 12, p. e0145122, 2015.

BERNDT, E. R.; COCKBURN, I. M. Price indexes for clinical trial research: a feasibility study. Monthly Labor Review, Washington, DC, p. 1-37, jun. 2014.

BRASIL. Ministério da Saúde. Resolução CNS/ MS n 251, de 5 de agosto de 1997. In: BRASIL. (Ed.). Resoluções do Conselho Nacional de Saúde. Brasília, DF: Ministério da Saúde, 2007. p. 371-377.

BRESSER-PEREIRA, L. C. Como sair do regime liberal de política econômica e da quaseestagnação desde 1990. Estudos Avançados, São Paulo, v. 31, n. 89, p. 7-22, 2017. 
DAL-RÉ, R. et al. Disclosure of investigators' recruitment performance in multicenter clinical trials: a further step for research transparency. PLoS Medicine, San Francisco, v. 8, n. 12, p. e1001149, 2011.

FLACCO, M. E. et al. Head-to-head randomized trials are mostly industry sponsored and almost always favor the industry sponsor. Journal of Clinical Epidemiology, Philadelphia, v. 68, n. 7, p. 811-820, 2015 .

GUPTA, A. et al. Clinical trial management of participant recruitment, enrollment, engagement, and retention in the SMART study using a Marketing and Information Technology (MARKIT) model. Contemporary Clinical Trials, Philadelphia, v. 42, p. 185-195, 2015.

HACKSHAW, A. A concise guide to clinical trial. Hoboken: Wiley-Blackwell; Chichester: BMJ Books, 2009.

ICH - INTERNATIONAL CONFERENCE ON HARMONISATION OF TECHNICAL REQUIREMENTS FOR REGISTRATION OF PHARMACEUTICALS FOR HUMAN USE. Guideline for good clinical practice $E 6$ (R1). Genebra: ICH, 1996. Disponível em: <https://bit.ly/2C9S5LV〉. Acesso em: 8 abr. 2017.

IVANOV, A. et al. Review and analysis of publication trends over three decades in three high impact medicine journals. PLoS One, Philadelphia, v. 12, n. 1, p. eo170056, 2017.

KOST, R. G. et al. A data-rich recruitment core to support translational clinical research. Clinical and Translational Science, Alexandria, v. 8, n. 2, p. 91-99, 2015.

KPMG LLP. Competitive alternatives 2016: KPMG's guide to international business locations costs. Vancouver: KPMG LLP, 2016. Disponível em: <https://bit.ly/1UBHH2I >. Acesso em: 20 mar. 2017.

MCDONALD, A. M. et al. Using a business model approach and marketing techniques for recruitment to clinical trials. Trials, London, v. 12, p. 74, 2011.

MURTHY, S.; MANDL, K. D.; BOURGEOIS, F. T. Industry-sponsored clinical research outside high-income countries: an empirical analysis of registered clinical trials from 2006 to 2013. Health Research Policy and Systems, London, v. 13, n. 1, p. 28, 2015.

PASCHOALE, H. S. et al. Clinical trials profile: professionals and sites. Contemporary Clinical Trials, Philadelphia, v. 31, n. 5, p. 438-442, 2010.

TREWEEK, S. et al. Methods to improve recruitment to randomised controlled trials: Cochrane Systematic Review and meta-analysis. BMJ Open, London, v. 3, n. 2, p. eoo236o, 2013.

VAN VALKENHOEF, G. et al. Deficiencies in the transfer and availability of clinical trials evidence: a review of existing systems and standards. BMC Medical Informatics and Decision Making, London, v. 12, p. 95, 2012.

VIERGEVER, R. F. et al. The quality of registration of clinical trials: still a problem. PLoS One, Philadelphia, v. 9, n. 1, p. e84727, 2014.

WATSON, J. M.; TORGERSON, D. J. Increasing recruitment to randomised trials: a review of randomised controlled trials. BMC Medical Research Methodology, v. 6, n. 1, p. 34, 2006. WOOD, A. J. J. Progress and deficiencies in the registration of clinical trials. New England Journal of Medicine, London, v. 36o, n. 8, p. 824-830, 2009. 